\title{
Evaluating the Aging of Multiple Emulsions Using Resonance- Enhanced Multiphoton Ionization Time-of-Flight Mass Spectrometry
}

\author{
Yukihiro TSUDA and Tomohiro UCHIMURA ${ }^{\dagger}$ \\ Department of Materials Science and Engineering, Graduate School of Engineering, University of Fukui, \\ 3-9-1 Bunkyo, Fukui 910-8507, Japan
}

\begin{abstract}
Resonance-enhanced multiphoton ionization time-of-flight mass spectrometry was applied to measurements of multiple emulsions with no pretreatment; a method for the quantitative evaluation of aging was proposed. We prepared water-inoil-in-water $(\mathrm{W} / \mathrm{O} / \mathrm{W})$ multiple emulsions containing toluene and $m$-phenylenediamine. The samples were measured immediately following both preparation and after having been stirred for $24 \mathrm{~h}$. Time profiles of the peak areas for each analyte species were obtained, and several intense spikes for toluene could be detected from each sample after stirring, which suggests that the concentration of toluene in the middle phase had increased during stirring. On the other hand, in the case of a W/O/W multiple emulsion containing phenol and $m$-phenylenediamine, spikes for $m$-phenylenediamine, rather than phenol, were detected after stirring. In the present study, the time-profile data were converted into a scatter plot in order to quantitatively evaluate the aging. As a result, the ratio of the plots where strong signal intensities of toluene were detected increased from $8.4 \%$ before stirring to $33.2 \%$ after stirring for $24 \mathrm{~h}$. The present method could be a powerful tool for evaluating multiple emulsions, such as studies on the kinetics of the encapsulation and release of active ingredients.
\end{abstract}

Keywords REMPI-TOFMS, multiple emulsion, aging

(Received February 29, 2016; Accepted April 6, 2016; Published July 10, 2016)

\section{Introduction}

An emulsion is a mixture of two immiscible liquids, such as oil and water, where one liquid is dispersed as droplets into another liquid. Emulsions are used in a wide range of applications, such as foods, cosmetics, and pharmaceuticals. Several collapse processes, e.g., creaming, flocculation, coalescence, and Ostwald ripening, are well known in emulsions, ${ }^{1-4}$ and other conditional changes, such as aging also occur. ${ }^{5}$ Therefore, evaluating emulsions is very important, and techniques such as small-angle X-ray scattering for measuring droplet sizes and conductivity measurement for revealing creaming rates have been employed. ${ }^{6-8}$

A multiple emulsion that is referred to as "emulsion of emulsion" has attracted increasing attention in recent years, and is a general term for emulsions that have several phases. For example, double emulsions, such as a water-in-oil-in-water (W/O/W) emulsion (Fig. 1) and an oil-in-water-in-oil (O/W/O) emulsion, consist of emulsion droplets that are smaller. For use in drug-delivery systems (DDS), which is one of the applications of multiple emulsions in the pharmaceutical field, active ingredients are expected to be encapsulated in an individual phase. ${ }^{9,10}$ However, aging as well as several collapse processes including migration, which is specific for multiple emulsions,

$\dagger$ To whom correspondence should be addressed.

E-mail: uchimura@u-fukui.ac.jp make it difficult to manage multiple emulsions in an expected form. The conditions and properties of multiple emulsions depend on factors such as constituents, concentrations, and preparation schemes.

Mass spectrometry is a powerful tool for analyzing constituents and their concentrations. Among mass spectrometric techniques, electrospray ionization quadrupole mass spectrometry (ESIQMS) is normally used to measure liquid samples. Until now, ESI-QMS has been used to measure surfactant-stabilized

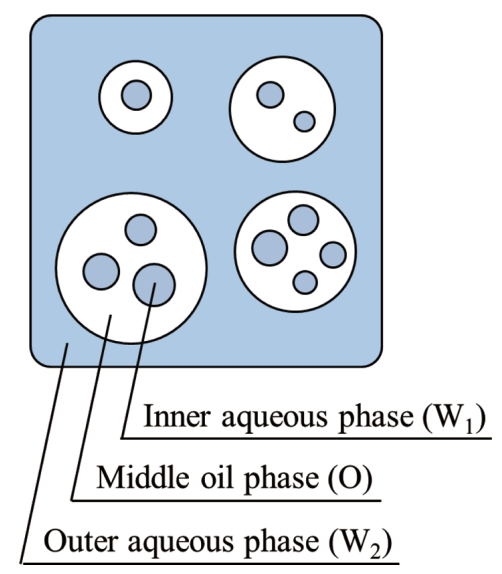

Fig. 1 Schematic representation of a W/O/W multiple emulsion. 
droplets containing small amounts of proteins. ${ }^{11}$ However, a direct analysis of emulsions is difficult via ESI because sample solutions require preparations, such as mixing with water or methanol, so that analytes will be efficiently ionized. In addition, the occurrence of multiply charged ions from several constituents with higher concentrations, typically several hundreds of $\mathrm{ng} / \mu \mathrm{L}$ or more, complicates the analysis. Moreover, QMS is a scanning-type MS, which is unsuitable for the simultaneous detection of several types of induced ions.

Recently, we achieved the direct analysis of constituents in oil-in-water $(\mathrm{O} / \mathrm{W})$ emulsions with neither pretreatment nor collection processes using resonance-enhanced multiphoton ionization time-of-flight mass spectrometry (REMPI-TOFMS). ${ }^{12}$ REMPI is a soft ionization method that has superior optical selectivity, and produces fewer contaminants. ${ }^{13-20}$ Moreover, TOFMS is not a scanning-type of MS, which has enabled us to detect all of the induced ions. TOFMS is also very robust against contamination, and is easy to clean because of the simple structure, even when there is contamination. Therefore, REMPI-TOFMS, either alone or combined with gas chromatography, is often used for the trace analysis of environmental samples. ${ }^{21-24}$ On the other hand, we have considered whether these advantages could also be suitable for the direct analysis of emulsions with higher concentrations of constituents. Therefore, a sample introduction technique for an emulsion sample towards REMPI-TOFMS has been developed, and the time profile of the analyte species was constructed by extracting the peak areas from a series of obtained mass spectra. ${ }^{12}$ In that report, several spikes appeared on the time profile of toluene, particularly when a milky white $\mathrm{O} / \mathrm{W}$ emulsion containing toluene as a dispersed phase was measured. We therefore concluded that the appearance of spikes indicated the existence of highly concentrated toluene in the form of small toluene droplets in the sample. We also reported the possibility that this method could be used for evaluating creaming of the emulsion. ${ }^{12}$ Moreover, we recently demonstrated the selective extraction of a hydrophobic compound of styrene to toluene small droplets in an emulsion by simultaneously detecting spikes from both toluene and styrene..$^{25}$

In the present study, REMPI-TOFMS was applied to the measurement of W/O/W multiple emulsions. We used two samples that contained either toluene or phenol as a hydrophobic compound and $m$-phenylenediamine as a hydrophilic compound, all of which can be ionized and detected by REMPI-TOFMS. Confirming the change in the obtained time profiles from the samples before and after stirring allowed estimations of the movement behaviors for each compound between the phases in W/O/W multiple emulsions. Moreover, we proposed a new method to quantitatively evaluate aging by analyzing a scatter plot constructed from the signal intensities of the obtained time profiles.

\section{Experimental}

\section{Reagents and sample preparation}

For preparation of $\mathrm{W}_{1} / \mathrm{O} / \mathrm{W}_{2}$ multiple emulsions, distilled water was used for both the inner phase $\left(\mathrm{W}_{1}\right)$ and the outer phase $\left(\mathrm{W}_{2}\right)$, and liquid paraffin (CAS No. 8042-47-5) was used as the middle phase $(\mathrm{O})$. Sorbitan monooleate (Span 80) and polyoxyethylene sorbitan monolaurate (Tween 20) were used as hydrophobic and hydrophilic surfactants, respectively. Toluene, phenol, and $m$-phenylenediamine were used as analyte species, the octanol-water partition coefficients $\left(\log K_{\text {ow }}\right)$ of which were $2.69,1.46$, and -0.33 , respectively. ${ }^{26-28}$ All chemicals were purchased from Wako Pure Chemical Industries (Osaka, Japan) with the exception of phenol, which was purchased from Kanto Chemical (Tokyo, Japan).

The present study employed a two-step emulsification procedure, which is a conventional preparation method for W/O/W multiple emulsions. ${ }^{29-31}$ Three types of solutions were prepared: a $m$-phenylenediamine aqueous solution, liquid paraffin containing toluene or phenol and Span 80, and a mixture of distilled water and Tween 20. The main fluids used in these solutions included distilled water for the inner aqueous phase (inner droplets), liquid paraffin for the middle oil phase (outer droplets), and distilled water for the outer aqueous phase. The solutions for the inner and middle phases were heated at $70^{\circ} \mathrm{C}$ for $10 \mathrm{~min}$ in a thermostatic bath. The solution for the inner phase was added to that for the middle phase in a 3:7 ratio (w/w), and subjected to a homogenizer (AS ONE, AHG-160D, Osaka) for $3 \mathrm{~min}$ at $10000 \mathrm{rpm}$ in order to prepare the inner emulsion. After homogenization, the resultant single emulsion was allowed to cool to room temperature. Then, the emulsion was added to the solution for the outer phase at a 1:9 ratio (w/w), and the obtained solution was stirred for $3 \mathrm{~min}$ at $1200 \mathrm{rpm}$. Hereafter, the obtained multiple emulsion will be referred to as a preparation sample (stirring time: $0 \mathrm{~h}$ ). We also prepared a multiple emulsion stirred at $1200 \mathrm{rpm}$ for $24 \mathrm{~h}$ after preparation. The final concentrations of the constituents in the multiple emulsions are mentioned in each figure caption; these solution volumes were calculated based on the weights of the reagents and their densities. Hereafter, the multiple emulsions containing toluene or phenol are referred to either as toluenemultiple emulsions or phenol-multiple emulsions.

\section{Apparatus}

The REMPI-TOFMS system has been described in detail elsewhere. ${ }^{12,25}$ A linear-type TOFMS, which is now commercially available (Hikari-GK, HGK-1, drift length $60 \mathrm{~cm}$, Fukuoka, Japan), was employed in the present study. We had developed a sample introduction technique using a pair of concentric capillaries for the measurement of simple emulsions, ${ }^{12}$ this system was applied to measurements of multiple emulsions. The pair of concentric capillaries consisted of two deactivated fused-silica capillary columns (GL Sciences, Tokyo). The lengths of the inner and outer capillaries were typically 55 and $35 \mathrm{~cm}$, respectively. The inner/outer diameters of both capillaries were $20 / 150$ and $320 / 450 \mu \mathrm{m}$, respectively. The tip of the inner column at the outlet side was adjusted to $c a .3 \mathrm{~mm}$ inward from the outer column. The capillary nozzle was heated to $40^{\circ} \mathrm{C}$ by raising the temperature of the flange. The inlet side of the inner capillary was held in a middle position, i.e., ca. $3 \mathrm{~cm}$ from the surface of a liquid sample and from the bottom of a 50-mL sample container. A sample was passed through an inner column. During a measurement, a multiple emulsion was gently stirred at $500 \mathrm{rpm}$ in order to prevent creaming. Ambient air was introduced from an outer column, and a flow meter (Kofloc, RK-1250, Kyoto, Japan) was used to adjust the flow rate at $2-3 \mathrm{~mL} / \mathrm{min}$. The resultant pressure in the chamber was $1 \times 10^{-2} \mathrm{~Pa}$.

The fourth harmonic of a pulsed Nd:YAG laser (Rayture Systems, GAIA II, $10 \mathrm{~Hz}, 266 \mathrm{~nm}, 4 \mathrm{~ns}$, Tokyo) was employed as an ionization laser. The energy of the laser pulses was adjusted to $20-50 \mu \mathrm{J}$, and the laser pulse was then focused with a plano-convex lens $(f=200 \mathrm{~mm})$. The ionization position was adjusted to $2 \mathrm{~mm}$ from the tip of an outer capillary. A digitizer (Agilent Technologies, Acqiris AP240, $1 \mathrm{GHz}$, $1 \mathrm{GS} / \mathrm{s}$ ) was used to record the signals of induced ions. Mass spectra using an average of 5 laser shots were recorded during 

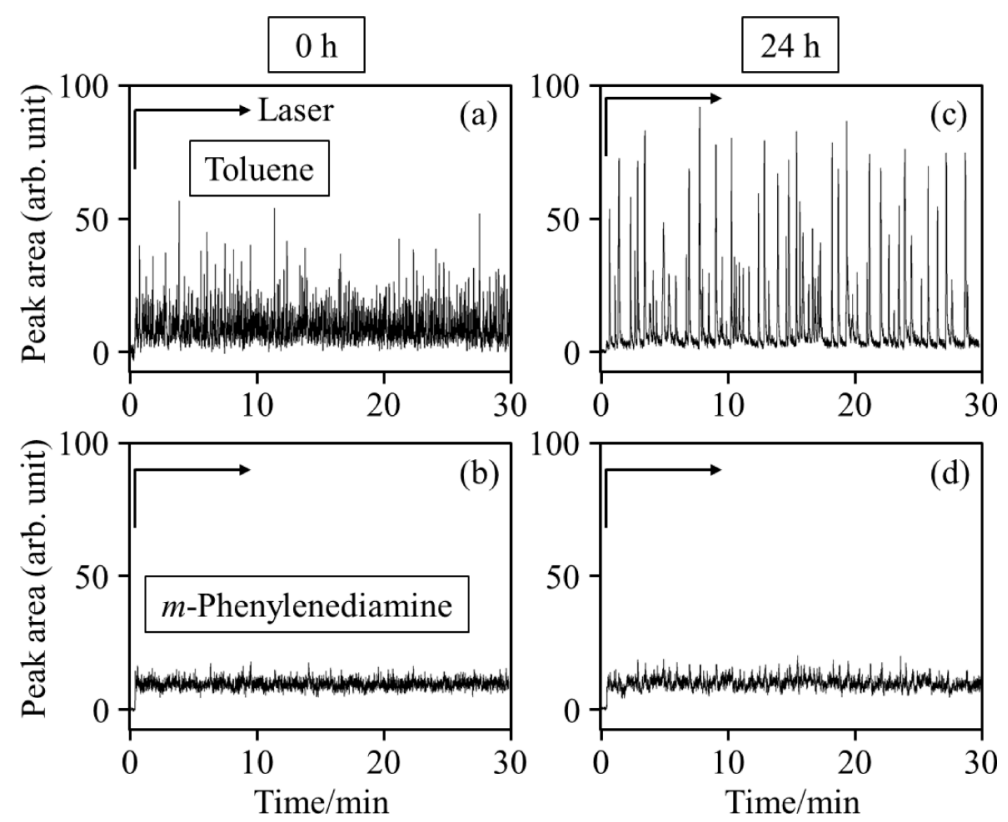

Fig. 2 Time profiles of the peak areas of toluene $(a, c)$ and $m$-phenylenediamine $(b, d)$ in a toluenemultiple emulsion. Stirring time: (a, b) $0 \mathrm{~h}$, (c, d) $24 \mathrm{~h}$. Concentration: $1200 \mathrm{ng} / \mu \mathrm{L}$ for toluene, $1200 \mathrm{ng} / \mu \mathrm{L}$ for $m$-phenylenediamine, $6000 \mathrm{ng} / \mu \mathrm{L}$ for Span $80,1200 \mathrm{ng} / \mu \mathrm{L}$ for Tween 20 . The laser beam for ionization was not introduced for the first $30 \mathrm{~s}$ from starting the recording in order to determine the baseline level.

the measurement in order to suppress the data volume derived from an extended period of recording. In the present study, the laser beam was not introduced for the first $30 \mathrm{~s}$ after starting the recording in order to determine a baseline level. The time profile of toluene was constructed by extracting the peak areas of both a molecular ion $(\mathrm{m} / \mathrm{z}, 92)$ and a fragment ion $(\mathrm{m} / \mathrm{z}, 91)$, which were equivalent to the results shown in Fig. 3 in the previous report, ${ }^{12}$ while the time profiles of phenol and $m$-phenylenediamine were constructed from the peak area of each molecular ion. The time profiles for both hydrophobic and hydrophilic compounds were obtained from the same measurement. The signal intensities for each time profile were normalized via the entire peak area obtained from each time profile.

In preliminary experiments, a multiple emulsion was aspirated through a capillary column connected to an aspirator (AS ONE, GAS-1), and the conditions of the samples passing through a capillary were observed using an inverted microscope (Olympus, IX51, ×10, NA 0.25, Tokyo).

\section{Results and Discussion}

\section{Time profiles of multiple emulsions}

Figure 2 shows the time profiles of toluene and $m$-phenylenediamine when measuring a toluene-multiple emulsion using REMPI-TOFMS. The results were obtained from the sample just after preparation (Figs. 2a and 2b) and after being stirred for $24 \mathrm{~h}$ (Figs. 2c and 2d).

Before the measurement, we estimated that toluene and $m$-phenylenediamine would be the main constituents in the middle oil phase $(\mathrm{O})$ and in the inner aqueous phase $\left(\mathrm{W}_{1}\right)$, respectively, of the $\mathrm{W}_{1} / \mathrm{O} / \mathrm{W}_{2}$ multiple emulsion just after preparation; that estimate was based on the terms of the sample preparation process and hydrophobicity. However, though spikes of toluene could be detected from the sample just after preparation (Fig. 2a), further intense spikes were also detected after stirring (Fig. 2c). On the other hand, spikes of $m$-phenylenediamine were not detected from the sample just after preparation (Fig. 2b), and though spikes were observed after stirring, their intensities were very small (Fig. 2d). Therefore, it was assumed that, contrary to our expectations, toluene and $m$-phenylenediamine were not present to an extreme degree in the middle and inner phases, respectively, but were present to some extent in an outer aqueous phase of the sample just after preparation. Furthermore, after stirring, several intense spikes were observed as a result of toluene, while the time profile of $m$-phenylenediamine was almost unchanged. The appearance of spikes indicated the existence of highly concentrated analyte species in the sample. ${ }^{12,25}$ In the present study, spikes of toluene should have been detected when highly concentrated toluene existed in outer droplets. Therefore, the concentration of toluene in the middle phase must have increased in the $\mathrm{W} / \mathrm{O} / \mathrm{W}$ multiple emulsion during stirring. The move from an outer aqueous phase was a possible reason. However, the concentration of $m$-phenylenediamine in the inner and outer phases seemed to have rarely changed either before or after stirring. These phenomena were a part of the aging of a multiple emulsion. As described later, we proposed a scatter plot in order to quantitatively evaluate the obtained results.

In the present study, almost all spikes obtained from the results of a toluene-multiple emulsion and a phenol-multiple emulsion (the latter is described later) consisted of several plots, while the previously reported spikes consisted of one plot. ${ }^{12,25}$ The spikes consisting of several plots were probably derived from the flow of an aggregate of small droplets and/or relatively large droplets through the capillary column. In a preliminary check, a toluenemultiple emulsion was stirred for several hours before being passed through a capillary column, such as the inner capillary used in the present study, connected to an aspirator; the condition 

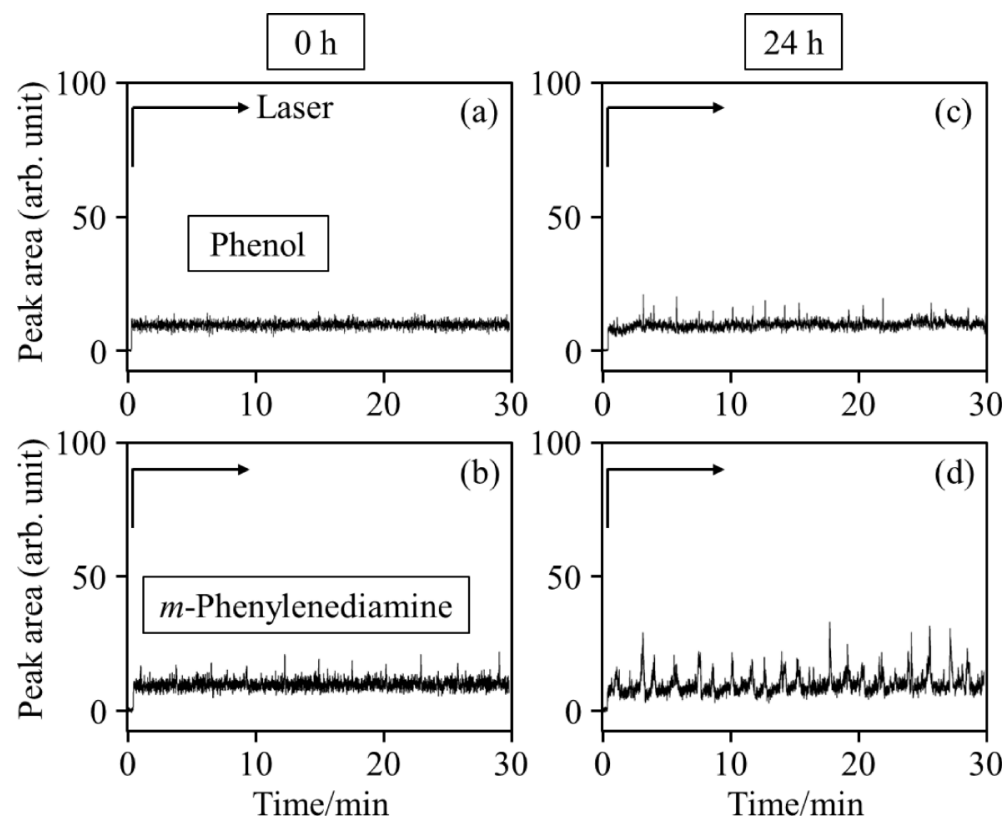

Fig. 3 Time profiles of the peak areas of phenol $(\mathrm{a}, \mathrm{c})$ and $m$-phenylenediamine $(\mathrm{b}, \mathrm{d})$ in a phenol-multiple emulsion. Stirring time: (a, b) $0 \mathrm{~h}$, (c, d) $24 \mathrm{~h}$. Concentration: $1100 \mathrm{ng} / \mu \mathrm{L}$ for phenol, $850 \mathrm{ng} / \mu \mathrm{L}$ for $m$-phenylenediamine, $6000 \mathrm{ng} / \mu \mathrm{L}$ for Span $80,1200 \mathrm{ng} / \mu \mathrm{L}$ for Tween 20 . The laser beam for ionization was not introduced for the first $30 \mathrm{~s}$ from starting the recording in order to determine the baseline level.

of the flow of the multiple emulsion was confirmed using an optical microscope. As a result, several aggregates were confirmed, where each aggregate consisted of small droplets, and a few seconds or less was required for the passage of a certain observation point. These results were appropriate for the number of plots needed for each spike in the time profile required by the use of REMPI-TOFMS.

The time profiles obtained from a phenol-multiple emulsion are shown in Fig. 3; these indicate behaviors that differ from those obtained from the toluene-multiple emulsion shown in Fig. 2. First, several spikes of $m$-phenylenediamine were observed from the time profiles of the phenol-multiple emulsion sample just after preparation and after stirring for $24 \mathrm{~h}$, which show the intensities becoming strong following stirring, but there were no phenol spikes just after preparation. Moreover, though several spikes were detectable by measuring the sample stirred for $24 \mathrm{~h}$, the intensities were considerably smaller than those of toluene, which were obtained from a toluene-multiple emulsion. These results suggest that the concentration of $m$-phenylenediamine in the inner phase was increased, but that of phenol in the middle phase, and possibly in the outer phase, was not dramatically changed during stirring. Incidentally, the width of the spikes of $m$-phenylenediamine obtained from the sample became wider after stirring, which suggested the possibility of the passage of further aggregated droplets through the capillary column. It was interesting that the width of the phenol spikes tended to be narrower. The reason for the difference in the width is unknown, but one conceivable explanation could be that there were two types of small droplets, i.e., phenol-rich droplets and $m$-phenylenediamine-rich droplets; these droplets aggregated with each other to form clusters where the number of the former droplets in each cluster might have been smaller.

\section{Scatter plots for the evaluation of aging}

The results from the aging of the multiple emulsions were obtained, as mentioned. Next, the phases of the analyte species were quantitatively evaluated by converting the time-profile data into a scatter plot.

Figure 4a shows a schematic illustration of the time profiles of the peak area of a hydrophobic compound, such as toluene, and a hydrophilic compound, such as $m$-phenylenediamine, in a $\mathrm{W}_{1} / \mathrm{O} / \mathrm{W}_{2}$ multiple emulsion; Fig. $4 \mathrm{~b}$ shows a scatter plot converted from the time profiles. In Fig. $4 b$, the horizontal and vertical axes show the peak areas of hydrophobic and hydrophilic compounds in each time profile, respectively. In the present study, the field in Fig. 4b was roughly classified into four groups, denoted as A to D. For instance, if the peak areas of both a hydrophobic and a hydrophilic compound were small for a given time profile, as shown in Fig. $4 \mathrm{a}$, and the spikes from the constituents could not be detected, the results were plotted in the field of A, as shown in Fig. 4b. This implies that both compounds are to some extent uniformly distributed, and/or the outer and inner droplets are very small so that a hydrophobic and a hydrophilic compound cannot be detected as a spike, even if each compound is encapsulated in the middle oil phase and an inner aqueous phase, respectively. In contrast, if both spikes appeared simultaneously, the result was plotted in the field of D. Corresponding examples of the conditions of each compound in each droplet in a $\mathrm{W}_{1} / \mathrm{O} / \mathrm{W}_{2}$ multiple emulsion are also depicted in Fig. 4b. Accordingly, the appearance of many plots in the area on the right, i.e., in $\mathrm{B}$ and $\mathrm{D}$, would indicate that a hydrophobic compound was encapsulated in the middle oil phase $(\mathrm{O})$, while a cluster in the upper area, i.e., in $\mathrm{C}$ and $\mathrm{D}$, would indicate that a hydrophilic compound was encapsulated in an inner aqueous phase $\left(\mathrm{W}_{1}\right)$. Although further discussion would be required in order to determine a threshold for the compartment of these fields, denoted as $a_{3}$ and $b_{3}$ in Fig. $4 \mathrm{~b}$, we tentatively decided on the following procedure. First, we 
(a)

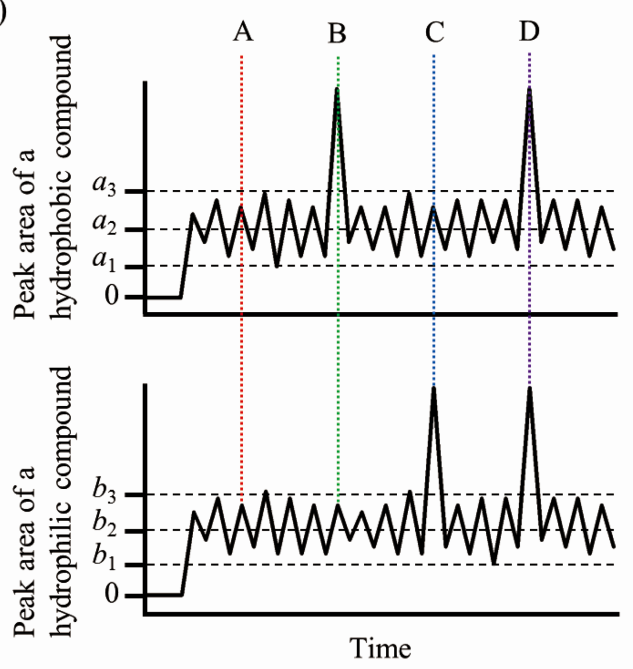

(b)
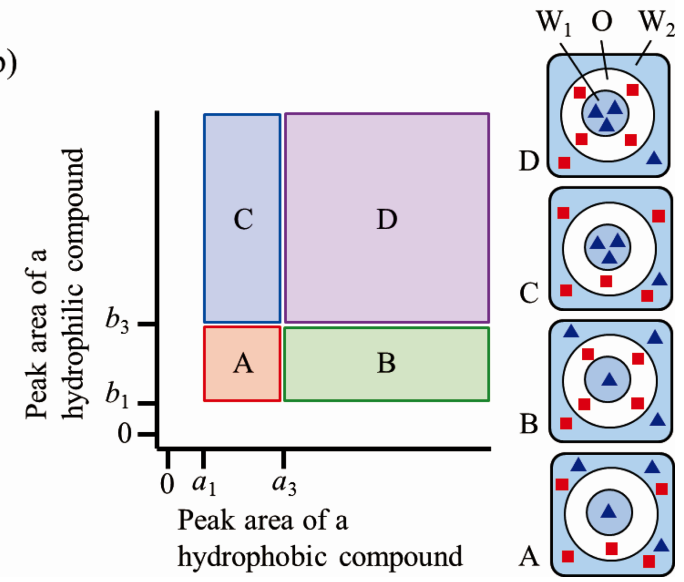

Fig. 4 Schematic representation of time profiles and a scatter plot (a) Time profiles of the peak areas of a hydrophobic and a hydrophilic compound in a $\mathrm{W}_{1} / \mathrm{O} / \mathrm{W}_{2}$ multiple emulsion, (b) schematic illustration of a scatter plot. The field was classified into four groups. Corresponding examples of the existence condition of each compound in each droplet are also shown. A: Both a hydrophobic compound ( and a hydrophilic compound $(\boldsymbol{\Delta})$ are to some extent uniformly distributed. B: A hydrophobic compound is mainly encapsulated in a middle oil phase $(\mathrm{O})$. C: A hydrophilic compound is mainly encapsulated in an inner aqueous phase $\left(\mathrm{W}_{1}\right)$. D: A hydrophobic and a hydrophilic compound are encapsulated in a middle oil phase $(\mathrm{O})$ and an inner aqueous phase $\left(\mathrm{W}_{1}\right)$, respectively.

confirmed the lowest signal intensity, $a_{1}$, from among the obtained data while introducing a laser beam from the time profile, as shown in Fig. 4a. Also, we roughly estimated the average of signal intensities, $a_{2}$, other than spikes. We then estimated a value for $a_{3}$ by adding the value of $a_{2}-a_{1}$ to $a_{2}$, i.e., $a_{3}=2 a_{2}-a_{1}$. Incidentally, the data of the baseline obtained when the laser beam for ionization would not irradiate are plotted at the lower left in Fig. 4b.

Figure 5 shows scatter plots converted from the time profiles of the toluene-multiple emulsion and the phenol-multiple emulsion shown in Figs. 2 and 3. In the case of the toluenemultiple emulsions shown in Figs. $5 \mathrm{a}$ and $5 \mathrm{~b}$, the plots were obviously shifted towards the right-hand side for the sample stirred for $24 \mathrm{~h}$, which means that the concentration of toluene in the middle phase increased after stirring. The ratio of the number of plots in $\mathrm{B}$ and $\mathrm{D}$ was $8.4 \%(=8.3 \%+0.1 \%)$ just after preparation, which was increased to $33.2 \%(=29.9 \%+$ $3.3 \%$ ) after stirring. In contrast, the ratio of the plots in A decreased. Interestingly, the area of A, which is the width in terms of the horizontal axis, is narrowed compared with those shown in Figs. 5a and 5b. One possible reason is that the concentration of toluene in the outer phase decreased, which resulted in a decrease in the signal intensities other than spikes for the time profile of toluene. Moreover, the ratios of the numbers of plots existing in $\mathrm{C}$ and $\mathrm{D}$ were slightly increased from $0.5 \%(=0.4 \%+0.1 \%)$ to $3.5 \%(=0.2 \%+3.3 \%)$ after stirring. These results suggest that the concentration of $m$-phenylenediamine in the inner phase slightly increased during stirring, which could not be quantitatively determined from the results of the time profiles shown in Figs. $2 b$ and $2 d$. Incidentally, a linear correlation with a positive slope between the two axes in Fig. 5b was found. The reason is unknown, but a plausible explanation is the presence of not only an individual outer droplet, but also the aggregates of the outer droplets in the capillary column; also, although the ratio of the concentrations between toluene and $m$-phenylenediamine was nearly the same among the droplets, the sizes of the outer droplets and/or the aggregates were all different.

In contrast, the change in the scatter plot of a phenol-multiple emulsion by stirring was considerably different from that of a toluene-multiple emulsion. First, plots were rarely shifted to the right-hand side (the ratio of the number of plots in B and D was only increased from 0.1 to $1.0 \%$ ), but were mainly shifted to the upper side (the ratio of the number of plots in $\mathrm{C}$ and $\mathrm{D}$ was increased from 1.5 to $17.6 \%$ ). These results once again suggest that the concentration of phenol in the middle and outer phases changed neither before nor after stirring, while that of $m$-phenylenediamine in the inner phase did. The difference in the degree of shift to the right-hand side between toluene and phenol seemed to derive from the difference in the partition coefficient $\left(\log K_{\text {ow }}=2.69\right.$ and 1.46, respectively), because the change of toluene to the middle oil phase occurred more easily than that of phenol due to hydrophobicity. Unfortunately, the reason that the concentration of $m$-phenylenediamine increased in the inner phase in the phenol-multiple emulsion remains unknown. When the partition coefficient $\left(\log K_{\mathrm{ow}}=-0.33\right)$ is taken into account, this compound should exist in the inner aqueous phase, rather than in the middle oil phase. Though a middle oil phase exists between the inner and outer phases, the miniaturization of droplets, which generally proceeds by stirring, ${ }^{29}$ might accelerate the moving of $m$-phenylenediamine from an outer phase to an inner phase and/or that of water from an inner phase to an outer phase. Though further discussion is required, the same tendency was also observed in the case of a toluene-multiple emulsion, i.e., the ratio of the number of plots in $\mathrm{C}$ and $\mathrm{D}$ was slightly increased from 0.5 to $3.5 \%$, as mentioned previously. In this manner, a scatter plot can reveal at which phases analyte species exist and, in addition, move with time. That is to say, the aging of multiple emulsions could be evaluated, which would be useful for both quality control and quality assurance. Moreover, the present method helps to explain how multiple emulsions change with time via encapsulating and releasing processes of ingredients that should be intimately associated with each other.

\section{Conclusions}

In the present study, we applied REMPI-TOFMS to measurements of multiple emulsions, and proposed a method for quantitative evaluations by constructing scatter plots using 

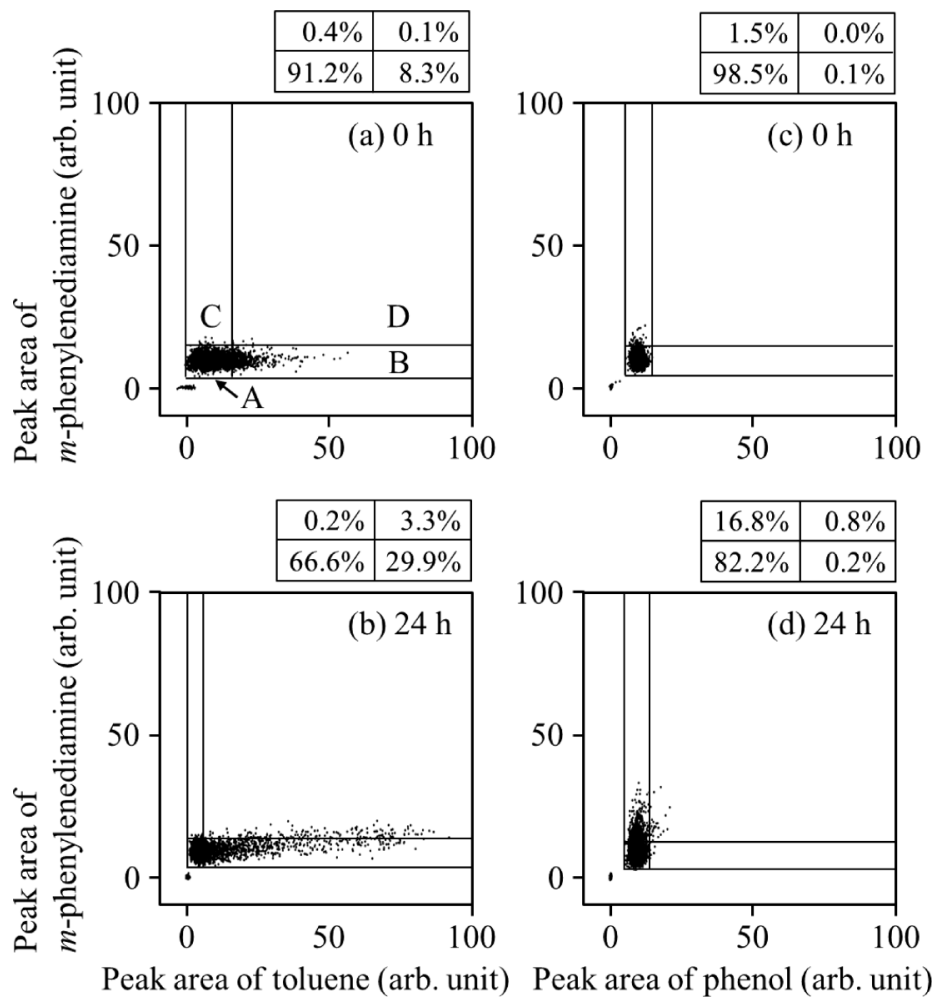

Fig. 5 Scatter plots obtained by measuring a toluene-multiple emulsion $(a, b)$ and phenol-multiple emulsion (c, d). Stirring time: (a, c) $0 \mathrm{~h},(\mathrm{~b}, \mathrm{~d}) 24 \mathrm{~h}$. The ratios of the number of plots obtained in each field against whole plots existing in $\mathrm{A}$ to $\mathrm{D}$ are also indicated.

the results of time profiles of the peak areas of the analyte species. The phases at which the analyte species existed in multiple emulsions as well as the moving of the species among phases before and after stirring were examined, though further study is required in order to divide the fields of the scatter plot. A two-step emulsification procedure that produces polydispersed emulsions was used for sample preparation in the present study. The construction techniques for monodispersed multiple emulsions are reported in the literature, ${ }^{32}$ and their use would enable us to discuss the aging process in greater detail. Studies on the kinetics of encapsulating and releasing active ingredients in multiple emulsions are gaining importance for future applications. REMPI-TOFMS can provide information about the local microenvironment in multiple emulsions without the need of pretreatment and collection processes. This method is therefore a powerful tool for the evaluation of aging as well as other collapse processes of emulsions.

\section{Acknowledgements}

This work was supported by a research grant provided by the JGC-S Scholarship Foundation.

\section{References}

1. K. Holmberg, B. Jönsson, B. Kronberg, and B. Lindman, "Surfactants and Polymers in Aqueous Solution", 2nd ed., 2003, John Wiley \& Sons, Chichester.

2. G. Vélez, M. A. Fernández, J. Muñoz, P. A. Williams, and R. J. English, J. Agric. Food Chem., 2003, 51, 265.
3. M. M. Robins, Curr. Opin. Colloid Interface Sci., 2000, 5, 265.

4. I. Capek, Adv. Colloid Interface Sci., 2004, 107, 125.

5. S. Matsumoto and M. Kohda, J. Colloid Interface Sci., 1980, 73, 13.

6. L. Auvray, J.-P. Cotton, R. Ober, and C. Taupin, J. Physique, 1984, 45, 913.

7. S. Arima, S. Ueno, A. Ogawa, and K. Sato, Langmuir, 2009, 25, 9777.

8. S. A. Gundersen and J. Sjöblom, Colloid Polym. Sci., 1999, 277,462 .

9. S. Farahmand, H. Tajerzadeh, and E. S. Farboud, Pharm. Dev. Technol., 2006, 11, 255.

10. D. J. McClements, E. A. Decker, and J. Weiss, J. Food Sci., 2007, 72, R109.

11. C. A. Smith, X. Li, T. H. Mize, T. D. Sharpe, E. I. Graziani, C. Abell, and W. T. S. Huck, Anal. Chem., 2013, 85, 3812.

12. H. Ishigami, Y. Tsuda, and T. Uchimura, Anal. Methods, 2014, 6, 5615.

13. D. M. Lubman and R. N. Zare, Anal. Chem., 1982, 54, 2117.

14. R. Zenobi, J.-M. Philippoz, P. R. Buseck, and R. N. Zare, Science, 1989, 246, 1026.

15. J. Matsumoto, C.-H. Lin, and T. Imasaka, Anal. Chem., 1997, 69, 4524.

16. R. Cohen, B. Brauer, E. Nir, L. Grace, and M. S. de Vries, J. Phys. Chem. A, 2000, 104, 6351.

17. J. Matsumoto, K. Misawa, S. Ishiuchi, and M. Fujii, Chem. Lett., 2009, 38, 74.

18. R. Zimmermann, Anal. Bioanal. Chem., 2013, 405, 6901.

19. A. Hamachi, T. Okuno, T. Imasaka, Y. Kida, and T. Imasaka, Anal. Chem., 2015, 87, 3027. 
20. T. Fujii and T. Uchimura, Anal. Methods, 2015, 7, 2611.

21. T. E. Hauler, U. Boesl, S. Kaesdorf, and R. Zimmermann, J. Chromatogr. A, 2004, 1058, 39.

22. B. K. Gullett, L. Oudejans, D. Tabor, A. Touati, and S. Ryan, Environ. Sci. Technol., 2012, 46, 923.

23. S. Sakurai and T. Uchimura, Anal. Sci., 2014, 30, 891.

24. S. Otto, T. Streibel, S. Erdmann, M. Sklorz, D. Schulz-Bull, and R. Zimmermann, Anal. Chim. Acta, 2015, 855, 60.

25. H. Fukaya, Y. Tsuda, and T. Uchimura, Anal. Methods, 2016, $8,270$.

26. C. T. Chiou, V. H. Freed, D. W. Schmedding, and R. L. Kohnert, Environ. Sci. Technol., 1977, 11, 475.

27. A. Leo, C. Hansch, and D. Elkins, Chem. Rev., 1971, 71,
525.

28. Material Safety Data Sheet, $m$-Phenylenediamine, MSDS No. EW160151, 2004, Wako Pure Chemical Industries, Ltd.

29. H. Okochi and M. Nakano, Adv. Drug Deliv. Rev., 2000, 45, 5.

30. F. Leal-Calderon, V. Schmitt, and J. Bibette, "Emulsion Science Basic Principles", 2nd ed., 2007, Springer, New York.

31. A. Aserin, "Multiple Emulsions Technology and Applications", 2008, John Wiley \& Sons, Hoboken, NJ.

32. L.-Y. Chu, A. S. Utada, R. K. Shah, J.-W. Kim, and D. A. Weitz, Angew. Chem. Int. Ed., 2007, 46, 8970. 